Research Paper

\title{
Clinical-pathological Characteristics and Prognostic Factors for Papillary Thyroid Microcarcinoma in the Elderly
}

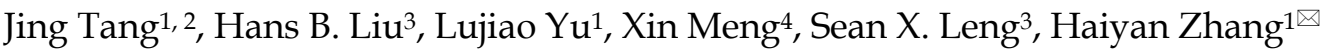 \\ 1. Department of Geriatrics, The First Hospital of China Medical University, Shenyang, Liaoning, 110001, P.R. China; \\ 2. Department of Geriatrics, The First People's Hospital of Jingmen, Jingmen, Hubei, 448000, P.R. China; \\ 3. Division of Geriatric Medicine and Gerontology, Department of Medicine, Johns Hopkins University School of Medicine, Baltimore, MD, USA; \\ 4. Department of Biochemistry and Molecular Biology, College of Basic Medical Sciences of China Medical University Shenyang, Liaoning, 110022, P.R. China. \\ $\square$ Corresponding author: Dr. Haiyan Zhang, Department of Geriatrics, the First Hospital of China Medical University, 155 North street Nanjing Road, \\ Shenyang, Liaoning, 110001, P.R. China. Tel: +86-024-83283698. E-mail: zhanghy@cmu.edu.cn. \\ (C) Ivyspring International Publisher. This is an open access article distributed under the terms of the Creative Commons Attribution (CC BY-NC) license \\ (https://creativecommons.org/licenses/by-nc/4.0/). See http://ivyspring.com/terms for full terms and conditions.
}

Received: 2017.09.05; Accepted: 2017.10.21; Published: 2018.01.01

\begin{abstract}
Background: The incidence of papillary thyroid microcarcinoma (PTMC) has increased dramatically over the past three decades worldwide. The annual rate of increase in the elderly $(\geq 65)$ PTMC patients is 1.4 times higher than that in the adult $(<65)$ PTMC patients. The aim of the present study is to identify the clinical-pathological characteristics and prognostic factors in the elderly PTMC patients.

Methods: The source population is PTMC patients whose information is available in the Surveillance, Epidemiology and End Results (SEER) database (2004-2013). We analyzed specific selected clinical-pathological parameters and prognostic factors for the PTMC patients who were aged 65 or above $(\mathrm{N}=4812)$.

Results: Within the elderly group, the male patients, in comparison to the females, had a higher percentage of lymph-node metastases $(5.29 \%$ vs. $12.27 \%, P<0.001)$, distant metastasis $(0.27 \%$ vs. $1.07 \%, P<0.001)$, and stage III-IV tumors $(9.19 \%$ vs. $15.85 \%, P<0.001)$. Moreover, the elderly patients had a lower median cause-specific survival (CSS) compared with the adult patients $(P<$ 0.001 ). Stage III-IV disease (hazard ratio (HR): $8.064, P<0.001$ ) was a strong risk factor for PTMC CSS. Being female (HR: 0.440,P=0.011), total thyroidectomy (HR: $0.057, P=0.001$ ), and lobectomy (HR: $0.058, P<0.001$ ) were all strong protectors of PTMC CSS.

Conclusion: Thyroidectomy improved CSS of the elderly PTMC patients. Compared with thyroid lobectomy, total thyroidectomy did not increase CSS for the elderly PTMC patients. The elderly PTMC patients who received radio therapy did not experience an increase in CSS.
\end{abstract}

Key words: papillary thyroid microcarcinoma; elderly patients; prognostic factors; SEER.

\section{Introduction}

Papillary thyroid carcinoma (PTC) is the most common malignant tumor of the thyroid gland. In the United States, the annual rate of increase in the number of PTC cases for the elderly (age 65 and above) is higher than that of the adults (between 18 and 65 years of age) (Annual percentage change (APC), $8.8 \%$ vs. $6.4 \%, P<0.001)^{[1]}$. As a subgroup of
PTC, Papillary thyroid microcarcinoma (PTMC) is defined as a PTC measuring $\leq 1 \mathrm{~cm}$ in maximum diameter [2]. Notably, the incidence of PTC has increased 3.2-fold from 1973 to 2006, primarily due to a rise in the incidence of PTMC, which has increased $441 \%$ over the past three decades ${ }^{[3-5]}$. Such an increase in the incidence of PTMC was attributed partly to the 
advances in sonography and fine-needle aspiration, both of which improved the detection of PTMC [6-8].

As of yet, it remains uncertain as to what the most effective treatment for PTMC is. Certain findings have suggested that most of PTMC patients have an excellent prognosis, thus suggesting that more extensive surgery and radiation therapy are unnecessary [9-11]. In contrast, there are other clinicians who recommend total thyroidectomy and radiation therapy, given that PTMC is associated with mortality, recurrence, and distant metastasis [12, 13]. Notably, that the therapeutic strategies for the elderly PTMC patients are much more complicated than those for the adults may be due to age-related physiological changes, limited social support, and comorbidities (such as cardiovascular diseases, cerebrovascular diseases, endocrine diseases, and lung diseases). Therefore, optimal treatment options for PTMC require weighing the risks and benefits of CSS associated with each therapy.

The PTMC patients ( $\geq 18$ years) who participated in this study were selected from the Surveillance, Epidemiology and End Results (SEER) database. Using these patients, we studied a number of selected clinical-pathological characteristics and treatment characteristics of the adult and elderly patients as well as the 5- and 10-year cancer-specific survival (CSS). We identified the prognostic factors that were associated with CSS in the elderly patients.

\section{Materials and Methods}

\section{Data source and study subjects}

We have conducted a retrospective cohort study using selected data from the SEER database. The database contains information regarding patient demographics, cancer incidence, primary characteristics of neoplasm, and CSS. All of this information was obtained from particular cancer registries from 18 geographic regions across the U.S., which together represents approximately $28 \%$ of the U.S. population.

The database used in this study was released on April 2016, and it was based on the data through the November 2013 SEER submission (1973-2013 varying). The PTC patients selected for our study included those who: 1) were diagnosed with PTC at some time from 2004 to 2013, inclusive, 2) were older than 18 years old (as of the time of PTC diagnosis), 3 ) had a tumor diameter of less than or equal to 1 centimeter, and 4) had active follow-up since the time of PTC diagnosis. Cases that had secondary malignancies and/or other primary malignancies were excluded from our study cohort. Based on this criterion, our study cohort consisted of a total of

\section{2,194 PTMC patients.}

PTMC cases were identified using the primary tumor site code of C739 (thyroid) along with the International Classification of Disease for Oncology, $3^{\text {rd }}$ edition (ICD-0-3) SEER site/histology validation list 2015 for PTC: 8050/3, 8260/3, 8350/3, 8340/3, $8341 / 3,8342 / 3,8343 / 3$, and 8344/3. Collaborative Stage (CS) Tumor Size: 000 (No mass/tumor found), 001-010 (001-010 millimeters (mm)), 990 (Microscopic focus or foci only and no size of focus given), 991 (described as "less than 1 centimeter $(\mathrm{cm}) ")$.

Demographic variables of interest to our study included gender, ethnicity, age, marital status, and insurance status; clinical data of interest included the status of surgery, radiotherapy, cause-specific death (CSD), and survival time (since PTC diagnosis until CSD, as of December 31, 2013, in months); pathological characteristics of interest included tumor size, histologic grade, as classified according to the American Joint Committee on Cancer (AJCC) 6th tumor-node-metastasis (TNM) as classified (6th edition, 2004).

With respect to surgery status, the cases were classified into four groups: "Unknown", "Total thyroidectomy", "No surgery", and "Lobectomy". The "Unknown group" included three subgroups "Thyroidectomy, Not otherwise specified (NOS)", "Surgery, NOS", and "Unknown if cancer-directed surgery performed, death certificate ONLY"; the "Total thyroidectomy" group consisted of two subgroups - "Subtotal or near total thyroidectomy" and "Total thyroidectomy"; "None; no cancer-directed surgery of primary site" was classified as "No surgery"; all other cases were clustered under the "Lobectomy" group. For insurance status, patients with "Any Medicaid", "Insured", or "Insured/No specifics" were clustered as the "Insured" group; for marital status, "Separated", "Divorced", "Single", and "Unmarried or Domestic Partner" were clustered as the "Single" group.

\section{Statistical methods}

All statistical analyses were performed using the Statistical Product and Service Solutions (SPSS) software for Windows 19.0 (SPSS Inc., Chicago, Illinois). The study subjects were stratified by age into two groups - adults $(<65)$ and the elderly $(\geq 65)$. Using the Chi squared $\left(\chi^{2}\right)$ test, selected clinical-pathological and treatment characteristics were compared between the two groups. CSS was determined by using the Kaplan-Meier method. The 5-year and 10-year CSS rates in elderly group were estimated from the survival curves, and the differences in CSS rates was determined by the 2-sided log-rank test. Cox 
proportional hazards analysis was performed to evaluate the risk factors of CSS in elderly group. All $P$ values were two-sided, and $P<0.05$ was considered to be statistically significant. Since the SEER database is publicly available and de-identified, our study was exempt from any approval from the institutional review board.

\section{Results}

\section{Patient clinical-pathological and treatment characteristics}

Our study cohort consisted of 32,194 PTMC patients whose data were derived from the SEER database, for the period between 2004 and 2013. Among these patients, there were 4,812 individuals in elderly group $(\geq 65)$ and 27,382 in the adult group $(<65)$. The comparison of selected clinical-pathological characteristics and treatment characteristics of the two groups is shown in Table 1. Compared with the adult group, the elderly had a larger proportion of males, more stage III-IV tumors, and more incidences of distant metastasis. The rates of total thyroidectomy, radioisotope therapy, and lymph-node metastases for the elderly were significantly lower than those for the adults (Table 1).

\section{The clinical-pathological characteristics of the elderly patients}

There were 1,123 (23.34\%) males and 3,689 (76.66\%) females in the elderly group. For the elderly PTMC patients, the comparison of selected clinical-pathological characteristics between genders is shown in Table 2. Lymph-node metastases was more common in males than in females $(12.27 \%$ vs. $5.29 \%, P<0.001)$. The percentage of distant metastasis in males was 5 times higher than that in females $(1.07 \%$ vs. $0.27 \%, P<0.001)$. There was also a higher percentage of males than females who had stage III-IV tumor $(15.85 \%$ vs. $9.19 \%, P<0.001)$.

\section{Survival differences}

As of December 31, 2013, the mean \pm standard deviation (range) period of follow-up was $45.82 \pm$ 32.22 (0 to 119) months. The 5- and 10-year CSS rate in the adult and elderly patients are shown in Table 3. Compared with the adults, the elderly patients had a significantly worse CSS $(P<0.001)$.

In the elderly patients, 40 disease-specific deaths $(0.83 \%)$ were observed, and a significant gender difference was observed in the 10-year CSS rate $(99.3 \%$ for females vs. $98.4 \%$ for males, $P<0.001)$ (Table 4).

On average, the patients who underwent thyroidectomy for PTMC had a better 10-year CSS rate compared to those who did not $(99.5 \%$ lobectomy, $99.1 \%$ total thyroidectomy vs. $93.1 \%$ no surgery, $P<0.001)$. Patients who underwent lobectomy and those who underwent total thyroidectomy had a similar 10-year CSS rate (Table 4).

Table 1. Comparison of clinical-pathological characteristics and treatment characteristics between the adult and elderly PTMC patients.

\begin{tabular}{|c|c|c|c|}
\hline Variable & $\begin{array}{l}<65 \text { years old }{ }^{1} \\
\mathrm{~N}=27,382(85.05 \%)\end{array}$ & $\begin{array}{l}\geq 65 \text { years old } 1 \\
\mathrm{~N}=4,812(14.95 \%)\end{array}$ & $P$ value $^{3}$ \\
\hline Gender & & & $<0.001$ \\
\hline Female & $22,829(83.40)$ & $3,689(76.70)$ & \\
\hline Male & $4,553(16.60)$ & $1,123(23.30)$ & \\
\hline Race & & & $<0.001$ \\
\hline White & $22,590(82.50)$ & 4,036 (83.90) & \\
\hline Black & $1,754(6.40)$ & $316(6.56)$ & \\
\hline Other ${ }^{2}$ & 3,038 (11.10) & $460(9.54)$ & \\
\hline Marital status & & & $<0.001$ \\
\hline Married & $18,170(66.36)$ & $28,17(58.54)$ & \\
\hline Unmarried & $7,691(28.09)$ & $1,717(35.68)$ & \\
\hline Unknown & $6,485(5.55)$ & $278(5.78)$ & \\
\hline Insurance status & & & $<0.001$ \\
\hline Insured & $20,316(80.60)$ & $3,771(78.40)$ & \\
\hline Uninsured & $581(1.60)$ & $21(0.40)$ & \\
\hline Unknown & $6,485(17.80)$ & $1,020(21.20)$ & \\
\hline Surgery & & & $<0.001$ \\
\hline No surgery & $143(0.52)$ & $29(0.60)$ & \\
\hline Lobectomy & $6,011(21.95)$ & $1,290(26.81)$ & \\
\hline Total thyroidectomy & $21,196(77.41)$ & $3,491(72.55)$ & \\
\hline Unknown & $32(0.12)$ & $2(0.04)$ & \\
\hline Radiotherapy & & & $<0.001$ \\
\hline Yes & $8,282(30.25)$ & $1,030(21.40)$ & \\
\hline No & $18,867(68.90)$ & $3,720(77.31)$ & \\
\hline Unknown & $233(0.85)$ & $62(1.29)$ & \\
\hline TNM stage & & & $<0.001$ \\
\hline I-II & $24,889(90.90)$ & $4,174(86.74)$ & \\
\hline III-IV & $1,953(7.13)$ & $517(10.74)$ & \\
\hline Unknown & $540(1.97)$ & $121(2.52)$ & \\
\hline $\mathrm{T}$ stage & & & 0.424 \\
\hline T1-T2 & $25,647(93.66)$ & $4,504(93.60)$ & \\
\hline T3-T4 & $1,677(6.12)$ & $302(6.28)$ & \\
\hline $\mathrm{T}_{\mathrm{x}}$ & $58(0.22)$ & $6(0.12)$ & \\
\hline $\mathrm{N}$ stage & & & $<0.001$ \\
\hline No & $23,826(87.01)$ & $4,388(91.19)$ & \\
\hline N1 & $3,235(11.82)$ & $344(7.15)$ & \\
\hline $\mathrm{N}_{\mathrm{x}}$ & $321(1.17)$ & $80(1.66)$ & \\
\hline M stage & & & $<0.001$ \\
\hline M0 & $27,033(98.73)$ & $4,737(98.44)$ & \\
\hline M1 & $58(0.21)$ & $22(0.46)$ & \\
\hline $\mathrm{M}_{\mathrm{x}}$ & 291(1.06) & $53(1.10)$ & \\
\hline Grade & & & $<0.001$ \\
\hline Well differentiated & $4384(16.01)$ & $118(2.45)$ & \\
\hline Moderately differentiated & $583(2.13)$ & $693(14.40)$ & \\
\hline Poorly differentiated & $43(0.16)$ & $12(0.25)$ & \\
\hline Undifferentiated & $6(0.02)$ & $5(0.10)$ & \\
\hline Unknown & $22366(81.68)$ & $3984(82.80)$ & \\
\hline \multicolumn{4}{|c|}{ PTMC, papillary thyroid microcarcinoma. } \\
\hline \multicolumn{4}{|c|}{ TNM, tumor-node-metastasis. } \\
\hline \multirow{2}{*}{\multicolumn{4}{|c|}{$\begin{array}{l}{ }^{1} \text { Refers to the age recorded at the time of thyroid papillary cancer diagnosis. } \\
\text { }{ }^{2} \text { Includes American Indian/AK Native, Asian/Pacific Islander. }\end{array}$}} \\
\hline & & & \\
\hline \multicolumn{4}{|c|}{$\mathrm{T}_{\mathrm{x}}$, primary tumor cannot be assessed. } \\
\hline \multicolumn{4}{|c|}{$\mathrm{N}_{\mathrm{x}}$, regional lymph nodes cannot be assessed. } \\
\hline \multicolumn{4}{|c|}{$\mathrm{M}_{\mathrm{x}}$, distant metastasis cannot be assessed. } \\
\hline $\begin{array}{l}{ }^{3} \text { Refers to comparison of the } \\
\text { elderly group. }\end{array}$ & differences in propo & rtions between th & adult and \\
\hline
\end{tabular}


Table 2. Comparison of selected clinical-pathological characteristics and treatment characteristics in female and male elderly PTMC patients.

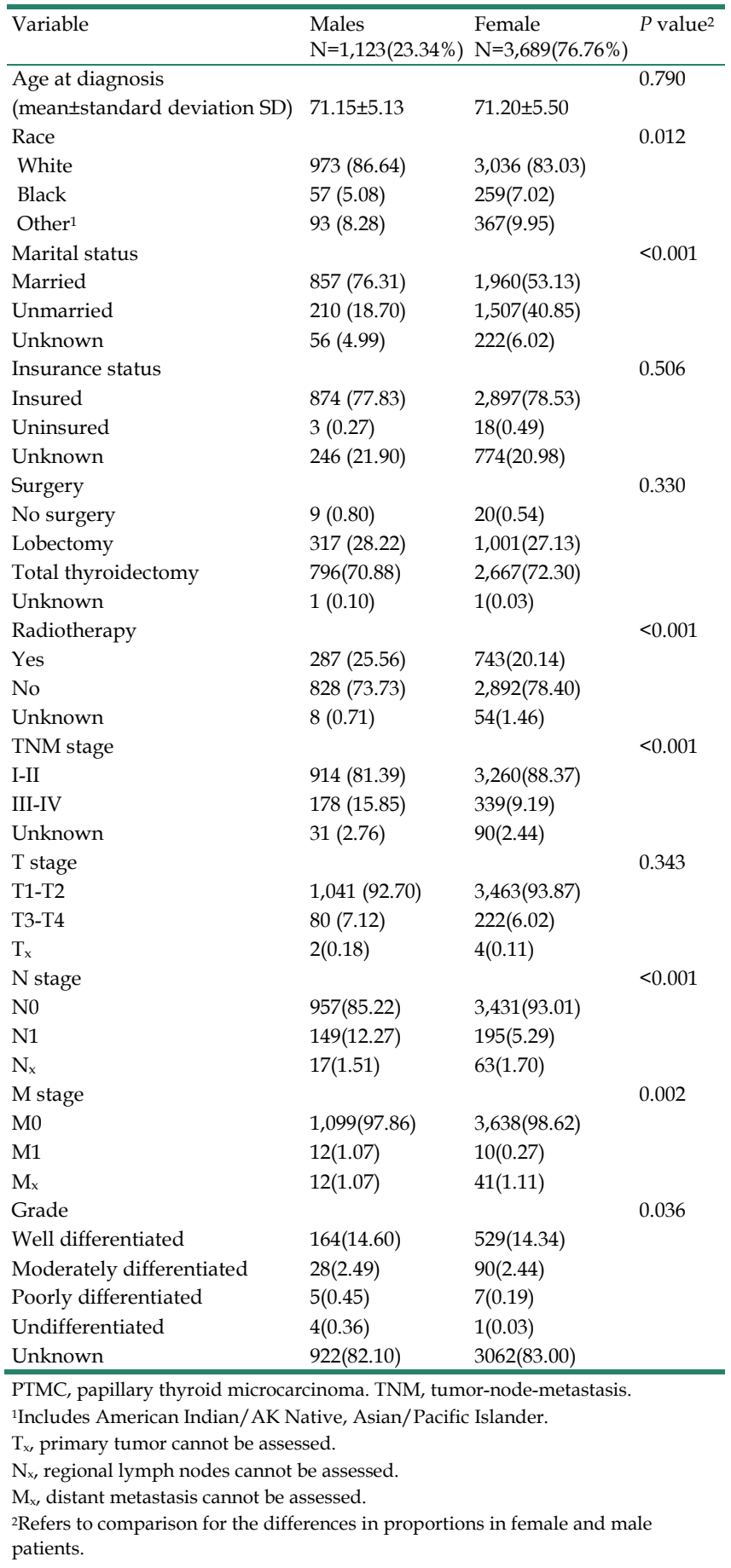

Table 3. Comparison of the 5- and 10-year CSS rate between the adult and elderly PTMC patients.

\begin{tabular}{lllll}
\hline & Death toll & 5-year CSS rate (\%) & Death toll & 10-year CSS rate (\%) \\
\hline$<65^{1}$ & 36 & 99.9 & 40 & 99.9 \\
$\geq 65^{1}$ & 34 & 99.3 & 40 & 99.2 \\
$\mathrm{P}$ value & $<0.001$ & & $<0.001$ \\
\hline
\end{tabular}

PTMC, papillary thyroid microcarcinoma.

CSS, cancer-specific survival.

${ }^{1}$ Refers to the age recorded at the time of thyroid cancer diagnosis.

${ }^{2}$ Refers to comparison of the differences in CSS between the adult and the elderly group.
On average, the 10-year CSS rate of patients who did not undergo radiotherapy was significantly higher than that of the patients who had radiotherapy $(99.5 \%$ vs. $97.9 \%, P<0.001)$ (Table 4$)$. Compared with the TNM stage I-II patients, those of stage III-IV had a lower 10-year CSS rate $(95.7 \%$ vs. $99.6 \%, P<0.001)$ (Table 4).

Table 4. The influence of specific prognostic factors on the CSS of PTMC patients, based on the log rank test.

\begin{tabular}{|c|c|c|c|c|c|}
\hline Variable & Number & $\begin{array}{l}5 \text {-year CSS } \\
\text { rate }(\%)\end{array}$ & P value & $\begin{array}{l}\text { 10-year CSS } \\
\text { rate }(\%)\end{array}$ & $P$ value \\
\hline Gender & & & $<0.001$ & & $<0.001$ \\
\hline Female & 1123 & 98.5 & & 98.3 & \\
\hline Male & 3685 & 99.5 & & 99.4 & \\
\hline Race & & & 0.312 & & 0.234 \\
\hline White & 4036 & 99.3 & & 99.1 & \\
\hline Black & 316 & 100 & & 100 & \\
\hline Other ${ }^{1}$ & 460 & 99.1 & & 98.9 & \\
\hline Marital status & & & 0.529 & & 0.417 \\
\hline Married & 2817 & 99.4 & & 99.3 & \\
\hline Unmarried & 1717 & 99.1 & & 99.0 & \\
\hline Unknown & 278 & 99.3 & & 99.3 & \\
\hline Insurance status & & & 0.932 & & 0.937 \\
\hline Insured & 3771 & 99.3 & & 99.3 & \\
\hline Uninsured & 21 & 100 & & 100 & \\
\hline Unknown & 1020 & 99.1 & & 98.6 & \\
\hline Surgery & & & $<0.001$ & & $<0.001$ \\
\hline No surgery & 29 & 93.1 & & 93.1 & \\
\hline Lobectomy & 1318 & 99.6 & & 99.5 & \\
\hline $\begin{array}{l}\text { Total } \\
\text { thyroidectomy }\end{array}$ & 3463 & 99.2 & & 99.1 & \\
\hline Unknown & 2 & 100 & & 100 & \\
\hline Radiotherapy & & & $<0.001$ & & $<0.001$ \\
\hline Yes & 1030 & 98.1 & & 97.9 & \\
\hline No & 3720 & 99.6 & & 99.5 & \\
\hline Unknown & 62 & 100 & & 100 & \\
\hline TNM stage & & & $<0.001$ & & $<0.001$ \\
\hline I-II & 4174 & 99.7 & & 99.6 & \\
\hline III-IV & 517 & 95.9 & & 95.7 & \\
\hline Unknown & 121 & 98.3 & & 98.3 & \\
\hline
\end{tabular}

\section{Univariate and multivariate analysis of risk factors for cancer-specific survival rates in the elderly PTMC patients}

Based on the univariate analysis, explanatory variables that were significantly associated with a lower 10-year CSS rate included the male gender $(P$ $<0.001)$, stage III-IV disease $(P<0.001)$, a lack of surgery $(P<0.001)$, and having undergone radiotherapy $(P<0.001)$, as shown in Table 5 .

Next, Cox proportional hazards regression models were utilized to quantify the prognostic significance of specific clinical-pathological characteristics and treatment characteristics, with adjustment to risk factors (Table 5). Variables that were determined to be associated with the 10-year 
CSS rate $(P<0.05)$ were included in the Cox proportional hazards regression model. Compared with the females, the males had a higher risk of developing CSD (hazard ratio (HR): 2.273, $P=0.011$ ). Notably, the elderly PTMC patients who underwent total thyroidectomy or lobectomy had a significantly lower risk for CSD compared to those who did not undergo any surgery for PTMC (HR:0.058 total thyroidectomy, $\mathrm{P}=0.001$; HR:0.057 lobectomy, $P<$ 0.001). Compared with thyroid lobectomy, total thyroidectomy did not significantly lengthen the CSS. Neither did radiotherapy translate to a survival benefit to the elderly patients (HR:1.647, $P>0.05$ ). The HR of TNM stage III-IV was 8.064 times higher than that of TNM stage I-II.

Table 5. Univariate and multivariate survival analyses, for various clinical-pathological characteristics and treatment characteristics of the elderly PTMC patients.

\begin{tabular}{|c|c|c|c|c|c|}
\hline \multirow[t]{2}{*}{ Variable } & \multirow[t]{2}{*}{ Number } & \multicolumn{2}{|c|}{ Univariate analysis } & \multicolumn{2}{|c|}{ Multivariate analysis } \\
\hline & & $\log \operatorname{rank} x^{2}$ test & $P$ value & $\mathrm{HR}(95 \% \mathrm{CI})$ & $P$ value \\
\hline Gender & & 13.906 & $<0.001$ & & \\
\hline Female & 1123 & & & Reference & \\
\hline Male & 3685 & & & $\begin{array}{l}0.440 \\
(0.234-0.828)\end{array}$ & 0.011 \\
\hline Surgery & & 28.259 & $<0.001$ & & \\
\hline No surgery & 29 & & & Reference & \\
\hline Lobectomy & 1318 & & & $\begin{array}{l}0.057 \\
(0.011-0.297)\end{array}$ & 0.001 \\
\hline $\begin{array}{l}\text { Total } \\
\text { thyroidectomy }\end{array}$ & 3463 & & & $\begin{array}{l}0.058 \\
(0.013-0.261)\end{array}$ & $<0.001$ \\
\hline Radiotherapy & & 23.282 & $<0.001$ & & \\
\hline Yes & 1030 & & & Reference & \\
\hline No & 3720 & & & $\begin{array}{l}0.620 \\
(0.282-1.310)\end{array}$ & 0.204 \\
\hline TNM stage & & 90.308 & $<0.001$ & & \\
\hline I-II & 4174 & & & Reference & \\
\hline III-IV & 517 & & & $\begin{array}{l}8.064 \\
(3.765-17.271)\end{array}$ & $<0.001$ \\
\hline
\end{tabular}

PTMC, papillary thyroid microcarcinoma

HR, hazard ratio

TNM, tumor-node-metastasis

\section{Discussion}

PTMC is a subgroup of PTC and accounts for about $30 \%$ of all PTC cases. Worldwide, the incidence of PTMC had risen rapidly over the past three decades. Along with the increasing incidence of PTMC, issues have also been raised concerning the reliability and validity of therapeutic strategies for the treatment of PTMC. Although some studies report that PTMC has a high rate of metastasis and therefore requires total thyroidectomy and radiation therapy [12, $14,15]$, no consensus has been reached on whether this treatment approach is optimal. Moreover, little is known about the prognostic significance of surgery type and radiation therapy in elderly PTMC patients. Mantinan et al. identified multi-focality as a significant risk factor for the recurrence rate of PTMC [16]. In contrast, a study by Shi et al. found that extra-thyroid invasion, lymph node metastases, and the BRAFV600E mutation were all major risk factors for PTMC [17]. Thus, the prognostic factors of elderly PTMC patients remains unclear. To address this, herein, we have identified a number of prognostic factors in the elderly PTMC patients.

We have found that, among our study population, the number of female PTMC patients was 4.6 times higher than that of the males, similar to the results of the previous studies $[6,18]$. To explain such a gender difference, it has been proposed that estrogen promotes the proliferation of thyroid cancer cells, thereby leading to a higher incidence in females as compared to males [19, 20].

Compared with the adult PTMC patients, the elderly PTMC patients has, in general, weaker health, more age-dependent physiological changes, as well as more complications. With this in consideration, clinicians generally recommended, for the elder patients, a more conservative treatment comprised of a lower rate of both total thyroidectomy and radiotherapy.

The 10 year-CSS rate for males and those with stage III-IV tumors are low compared to that of females and those with stage I-II tumors, respectively. Thus, that the 10-year CSS rate in the elderly patients was significantly lower than that of the adults can be potentially be attributed to the fact that the elderly PTMC patients has a higher percentage of stage III-IV tumors as well as a higher percentage of males.

This study has also found that the risk of CSD in the male patients was 2.273 times higher than that in the females, similar to the findings by Lin et al. [21]. Hsieh et al. [22] reported that most elderly male patients in the late stages of the disease were prone to developing lymph node metastasis and distant metastasis, both of which resulted from the levels of sex hormone and unhealthy behavior (such as smoking and drinking). Consistent with such findings, herein, we have found that the 10-year CSS rate for the elderly males was significantly lower than that of the elderly females, and that gender is an independent prognostic factor for PTMC in the elderly patients.

Thus far, no consensus has been reached on whether PTMC patients should undergo surgery, and the impact of surgery on the long-term CSS also remains controversial [11, 12, 23-25]. Herein, we have found that the 10-year CSS rate of the PTMC patients who did not undergo surgery was lower than that of the patients who underwent surgery. Furthermore, among the patients who underwent surgery for PTMC, the 10-year CSS rate of patients who had 
lobectomy was slightly higher than that of the patients who underwent total thyroidectomy. In addition, we have found that surgery was an independent risk factor of prognosis for the elderly patients with PTMC, but that the surgery type was not. Compared with total thyroidectomy, lobectomy reduces the incidence of post-surgery complications (such as permanent hypoparathyroidism, recurrent laryngeal nerve palsy, and hypocalcemia), and the remaining partial thyroid tissue that contributes to the recovery of thyroid function. In sum, in order to avoid excessive treatment, the choice of treatment for PTMC in the elderly patients should account for all the relevant risk factors.

McLeod et al. [26] had shown that the use of ${ }^{131}$ I radioactive iodine after low-risk PTC surgery did not significantly reduce the risk of recurrence or mortality. In contrast, Schvartz et al. [27] had found that radiotherapy reduced the overall survival of low-risk differentiated thyroid cancer (DTC) $(94.6 \%$ vs. $95.8 \%, P<0.001)$ and that radiotherapy was not an independent prognostic risk factor of DTC. Our study has demonstrated that the 10-year CSS rate in the elderly PTMC patients who underwent radiation therapy for PTMC was significantly lower than that of the elderly PTMC patients who did not undergo such therapy. In spite of this, we have found that radiotherapy was not an independent prognostic factor in the elderly patients with PTMC. Overall, in order to reduce the physical and mental burden of the elderly patients and improve their quality of life, comprehensive assessment of the conditions of elderly patients should be conducted to determine whether radiotherapy is warranted.

A number of studies have demonstrated that both TNM stage and PTMC recurrence rate are associated with the risk of lymph node metastasis in PTMC patients [28, 29]. Consistent with these findings, we have found that TNM stage was an independent prognostic factor in elderly patients with PTMC, and that the 10-year CSS rate of stage III-IV elderly patients was lower than that of the elderly patients in stage I-II. Although certain studies suggest that the scope of surgery for PTMC patients should be expanded to improve the survival rate of the PTMC patients, no consensus has been reached [29].

Race has been showed to be independent prognostic factors in lots of malignant diseases using SEER database ${ }^{[30]}$, but it had no impact on survival in our study. One of the possible reasons is that thyroid cancer patients have pretty good prognosis, especially for those who are already older than 65 years old. The 5 year cause specific survival was $99.1 \%$ and $100 \%$ for Caucasian and African American.

It is worth noting that the SEER database does not include information on certain risk factors, including endocrine therapy, local recurrence, and other complications (The elderly patients had more complications), all of which might have an impact on the prognosis of the elderly patients with PTMC. Moreover, TSH, T4 and T3 are not available in the SEER database, we have no access to get these data.

In conclusion, gender, surgery, and TNM stage were all independent prognostic factors for elderly patients with PTMC. For males, patients who have not underwent surgery for PTMC, and patients with stage III-IV tumors, the prognosis was relatively poor. Radiotherapy did not lead to an improvement in the 10 -year CSS rate in the elderly patients with PTMC. In addition, the various types of surgery did not differ with respect to the ability to improve the 10-year CSS. As such, in order to reduce the risk of recurrence, avoid over-treatment, and extend the lifespan of the elderly PTMC patients, all the risk factors and the specific condition of each patient must be carefully assessed in the determination of treatment strategies.

\section{Abbreviations}

AJCC, American Joint Committee on Cancer; $\mathrm{APC}$, annual percentage change; $\mathrm{CI}$, confidence interval; CS, Collaborative Stage; CSD, cause-specific death; CSS, cause-specific survival; DTC, differentiated thyroid cancer; HR, hazard ratio; ICD-O, International Classification of Diseases for Oncology; PTC, papillary thyroid carcinoma; PTMC, papillary thyroid microcarcinoma; SEER, Surveillance, Epidemiology, and End Results; TNM, Tumor-Node-Metastasis.

\section{Acknowledgments}

We would like to thank the staff of the National Cancer Institute and their colleagues across the United States, and the Information Management Services, Inc., all who have been involved with the SEER Program.

\section{Funding}

This work was supported by the National Natural Science Foundation of China (Grant number: 81301838, 81271292, 81572831) and funding from the Irma and Paul Milstein Medical Asian American Partnership (MMAAP) Foundation Program for Senior Health fellows, supported by the MMAAP Foundation (http://www.mmaapf.org), to Dr. Haiyan Zhang.

\section{Competing Interests}

The authors have declared that no competing interest exists. 


\section{References}

1. Morris LG SA, Tosteson TD. The increasing incidence of thyroid cancer: the influence of access to care. Thyroid. 2013;(23): 885-891.

2. Christoph HE, Leslie H. Sobin. The WHO Histological Classification of Thyroid Tumors: A Commentary on the Second Edition. Cancer. 1989;63: 908-911.

3. Hay ID, Hutchinson ME, Gonzalez-Losada T, et al. Papillary thyroid microcarcinoma: a study of 900 cases observed in a 60-year period. Surgery. 2008;144: 980-987; discussion 987-988.

4. Simard EP, Ward EM, Siegel R, et al. Cancers with increasing incidence trends in the United States: 1999 through 2008. CA Cancer J Clin. 2012;62: 118-128.

5. Cramer JD, Fu P, Harth KC, et al. Analysis of the rising incidence of thyroid cancer using the Surveillance, Epidemiology and End Results national cancer data registry. Surgery. 2010;148: 1147-1152; discussion 1152-1143.

6. Roti E, Degli Uberti EC, Bondanelli M, et al. Thyroid papillary microcarcinoma: a descriptive and meta-analysis study. Eur J Endocrinol. 2008;159: 659-673.

7. Enewold L, Zhu K, Ron E, et al. Rising thyroid cancer incidence in the United States by demographic and tumor characteristics, 1980-2005. Cancer Epidemiol Biomarkers Prev. 2009;18: 784-791.

8. Hoang JK, Choudhury KR, Eastwood JD, et al. An exponential growth in incidence of thyroid cancer: trends and impact of CT imaging. AJNR Am J Neuroradiol. 2014;35: 778-783.

9. Yu XM, Wan Y, Sippel RS, et al. Should all papillary thyroid microcarcinomas be aggressively treated? An analysis of 18,445 cases. Ann Surg. 2011;254: 653-660.

10. Haymart MR, Cayo M, Chen H. Papillary thyroid microcarcinomas: big decisions for a small tumor. Ann Surg Oncol. 2009;16: 3132-3139.

11. Wang TS, Goffredo P, Sosa JA, et al. Papillary thyroid microcarcinoma: an over-treated malignancy? World J Surg. 2014;38: 2297-2303.

12. Chow SM, Law SC, Chan JK, et al. Papillary microcarcinoma of the thyroid-Prognostic significance of lymph node metastasis and multifocality. Cancer. 2003;98: 31-40

13. Page C, Biet A, Boute P,et al . Aggressive papillary' thyroid microcarcinoma. Eur Arch Otorhinolaryngol. 2009;266: 1959-1963.

14. Moon HJ, Kim EK, Yoon JH, et al. Clinical implication of elastography as a prognostic factor of papillary thyroid microcarcinoma. Ann Surg Oncol. 2012;19: 2279-2287.

15. Creach KM, Siegel BA, Nussenbaum B, et al. Radioactive iodine therapy decreases recurrence in thyroid papillary microcarcinoma. ISRN Endocrinol. 2012;2012: 816386

16. Mantinan B, Rego-Iraeta A, Larranaga A, et al. Factors influencing the outcome of patients with incidental papillary thyroid microcarcinoma. J Thyroid Res. 2012;2012: 469397.

17. Shi C, Guo Y, Lv Y, et al. Clinicopathological Features and Prognosis of Papillary Thyroid Microcarcinoma for Surgery and Relationships with the BRAFV600E Mutational Status and Expression of Angiogenic Factors. PLoS One. 2016;11: e0167414.

18. Kuo EJ, Goffredo P, Sosa JA, et al. Aggressive variants of papillary thyroid microcarcinoma are associated with extrathyroidal spread and lymph-node metastases: a population-level analysis. Thyroid. 2013;23: 1305-1311.

19. Manole D, Schildknecht B, Gosnell B, et al. Estrogen promotes growth of human thyroid tumor cells by different molecular mechanisms. J Clin Endocrinol Metab. 2001;86: 1072-1077.

20. Zeng Q, Chen G, Vlantis A, et al. The contributions of oestrogen receptor isoforms to the development of papillary and anaplastic thyroid carcinomas. J Pathol. 2008;214: 425-433.

21. Lin DZ, Qu N, Shi RL, et al. Risk prediction and clinical model building for lymph node metastasis in papillary thyroid microcarcinoma. Onco Targets Ther. 2016;9: 5307-5316.

22. Hsieh SH, Chen ST, Hsueh C, et al. Gender-Specific Variation in the Prognosis of Papillary Thyroid Cancer TNM Stages II to IV. Int J Endocrinol. 2012;2012: 379097.

23. Ito $\mathrm{Y}$, Miyauchi A, Kihara M, et al. Patient age is significantly related to the progression of papillary microcarcinoma of the thyroid under observation. Thyroid. 2014;24: 27-34.

24. Megwalu UC. Observation versus thyroidectomy for papillary thyroid microcarcinoma in the elderly. J Laryngol Otol. 2016: 1-4.

25. Lin HW, Bhattacharyya N. Survival impact of treatment options for papillary microcarcinoma of the thyroid. Laryngoscope. 2009;119: 1983-1987.

26. McLeod DS, Sawka AM, Cooper DS. Controversies in primary treatment of low-risk papillary thyroid cancer. Lancet. 2013;381: 1046-1057.

27. Schvartz C, Bonnetain F, Dabakuyo S, et al. Impact on overall survival of radioactive iodine in low-risk differentiated thyroid cancer patients. J Clin Endocrinol Metab. 2012;97: 1526-1535.

28. Kuo SF, Chao TC, Chang HY, et al. Prognostic evaluation of patients with multicentric papillary thyroid microcarcinoma. J Formos Med Assoc. 2011;110: 511-517.

29. Xu JM, Xu HX, Li XL, et al. A Risk Model for Predicting Central Lymph Node Metastasis of Papillary Thyroid Microcarcinoma Including Conventional Ultrasound and Acoustic Radiation Force Impulse Elastography. Medicine (Baltimore). 2016;95: e2558.

30. Lin JZ, Qiu MQ, Xu RH, et al. Comparison of survival and clinicopathologic features in colorectal cancer among African American, Caucasian, and Chinese patients treated in the United States: Results from the surveillance epidemiology and end results (SEER) database. Oncotarget. 2015; 6: 33935-33943. 